\title{
Strain measurement and determining coefficient of plastic anisotropy using digital image correlation (DIC)
}

\author{
N. Hedayati, R. Madoliat and R. Hashemia \\ School of Mechanical Engineering, Iran University of Science and Technology, Tehran, Iran
}

Received 16 April 2016, Accepted 24 Octobre 2016

\begin{abstract}
The deformation and strain measurements have always been one of the main issues in structural engineering. Measuring displacements accurately without damaging structures are an important aim in experimental analysis. For an example, the existing structures need to be evaluated whether they are still fit for purposes or not. To assess complex structures, we need an acceptable and enough amount of data Current strain measurement technologies are not capable of evaluating compounded structures. This paper investigates on an alternative method that uses digital images to provide a full displacement and strain field called Digital Image Correlation (DIC); This technique compares the digital images taken from digital cameras, before and after deformation. We also calculated the coefficient of plastic anisotropy. An aluminium 1050 specimen was loaded in tension according to ASTM E8 testing method while the Sony camcorder captured the video of the specimen being loaded. Once the video was captured, the images were snapshot from video strip using a built-in function of the camera. After that, the images were processed using the Matlab software. The results determined by the extensometer and the optical strain measurement method were compared to each other. This comparison shows that if the test's setup could be configured in a good way, the error will be less than $2 \%$.
\end{abstract}

Key words: Strain measurement / digital image correlation / tensile test / finite element / coefficient of plastic anisotropy

\section{Nomenclature}

\begin{tabular}{|ll|}
\hline$u$ & x displacement \\
$v$ & y displacement \\
$J$ & Jacobian of mapping function \\
$\epsilon_{l}$ & Length strain \\
$\epsilon_{w}$ & Width strain \\
$\nabla$ & Gradient \\
$\epsilon_{x x}$ & Small-strain in $x$ direction \\
$\epsilon_{y y}$ & Small-strain in $y$ direction \\
\hline
\end{tabular}

\section{Introduction}

Digital image correlation is an optical technique based on the comparison of images before, during and after the deformation of a test specimen, usually captured from a digital camera. The figures are split into a series of investigation blocks or subsets, including a limited number

\footnotetext{
a Corresponding author: rhashemi@iust.ac.ir
}

of pixels. DIC uses a correlation schema to catch the displacements by recognizing subsets of relevant grey extent quantity between the speckle pattern in each subset of the deformed and undeformed figures. The location where the correlation function value is maximized in the deformed image corresponds to the movement of the pattern during deformation. Different criteria are defined for correlation in the literature; these criteria can be classified into two groups, CC criteria and SSD correlation criteria [1,2]. To facilitate the correlation a stochastic speckle pattern is applied to the specimen surface to provide random grey level variations, the quality of which is fundamental to the precision of the measured displacement data. Spatial accuracy of the data is enlarged by decreasing the extent of the subsets, but as the investigation cell size decreases, the uncertainty in the strain measurement increases because of a decline in the number of characteristics to track within the subset [3].

The Digital Image Correlation method has been used in the literature since the beginning of 1980 s $[4,5]$ and takes different kinds of form. The method which will be concentrated in this paper, is known as two dimensional DIC and includes the usage of an individual camera to 


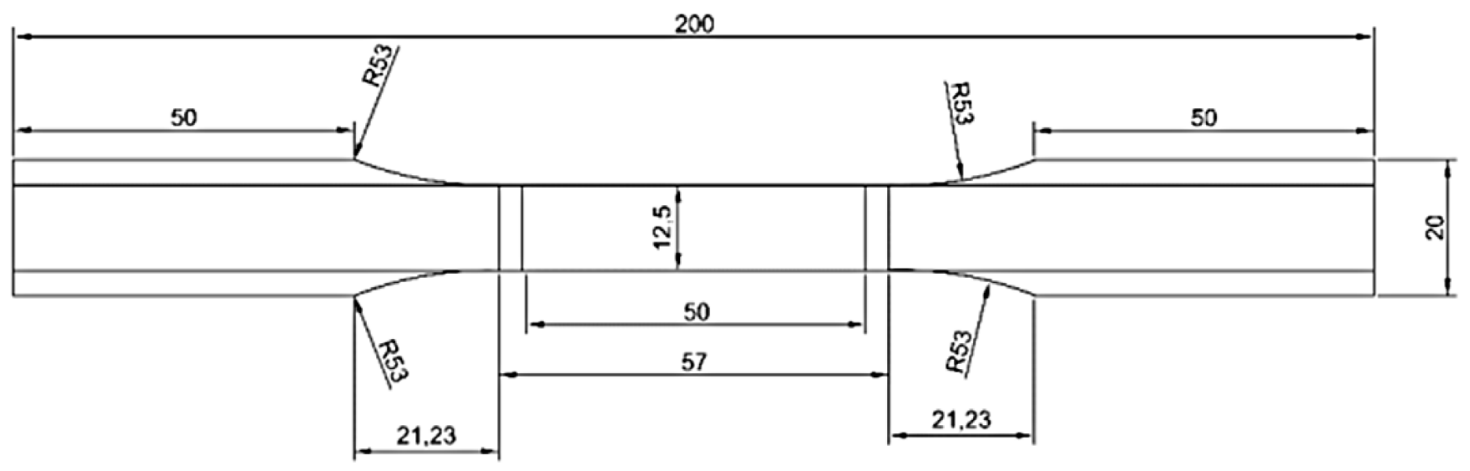

Fig. 1. The shape and measure of the aluminium specimen.

capture displacements in a plane [6]. This method has the privilege that it only needs a single camera, thus decreasing the equipment expenses and allowing for additional measurement regions to be added without needing these measurement regions to overlap. However, it has one significant disadvantage and that is all the displacements should befall within a single motionless measurement plane otherwise inaccurate results will be obtained. Researchers have dominated this constraint by presenting 2.5-D [7] and 3-D DIC systems [8] that use several cameras to measure a $3-\mathrm{D}$ displacement field. These establishments tend to be more costly and specialized and therefore are not widely usable in the structural engineering research societies. Many researchers have previously used DIC approach to measure one dimensional strains for images produced with softwares or experimentally, with different situations of success. Smith et al. [9] found $100 \mu \varepsilon$ deviances in strain measurement which is standard. They used concentric lens, which decreases the out of plane inflection effects, but the resolution of their camera $(640 \times 480$ pixels $)$ plus bicubic spline subpixel interpolation code have been affected their accuracy in measurement negatively. The errors produced in displacement when using the DIC method, are deductions of a pixel commonly and while evaluating the strain, the consequence of this error can be decreased via applying a larger longitude for gage length (in pixels) to produce a smaller error in strain measurement. Therefore cameras with higher resolution increase the accuracy of measurement by permiting the larger amount of gage lengths. Wattrisse et al. [10] obtained mean strain errors of $210 \mu \varepsilon$. They used a camera with lower resolution $(1317 \times 1035$ pixels $)$ in addition to a lower accurate subpixel interpolation code (a bicubic spline). Also, Wattrisse et al. employed a lens which had $55 \mathrm{~mm}$ focal length. One of the most important factors for minimizing the error in strain measurement is the focal length of lens; larger focal lengths results in smaller errors. Hung and Voloshin [11] carried out a single axial test on a sheet made of aluminum and achieved an average of $5.8 \%$ error. They didn't give any specifics on the experimental equipments and subpixel interpolation code; so we cannot evaluate the possible causes which led to error, compared to their offered method. Comparing to other tech- niques for measuring deformation, one notable privilege of the two dimensional DIC is that fewer requirements are needed and implementation is readily done with an ordinary setup. Anyhow, this does not mean that the accuracy of measurement in 2D DIC is not altered by the measuring mechanism at all. Oppositely, it is figured on that the accuracy of displacement and strain measurement in 2D DIC relies intensely on the condition of the loading mechanism, the integrity of the image capturing system and the choice of a specific algorithm for correlation [12]. Some of the errors and their possible causes are presented in Table 1. It is obvious that errors assessment related to different situations is significant in order to find procedures to make an improvement in measurement accuracy of 2D DIC [13]. Evaluating the suitability of strain measurement of aluminium by MATLAB GUIs, comparing the results obtained by DIC with those obtained by a conventional mechanical transverse extensometer, and obtaining the coefficient of plastic anisotropy, are the main objectives of this paper.

\section{Methods}

\subsection{Specimen preparation}

In this paper, the tensile tests for the aluminium samples were conducted in accordance with ASTM E8. By referring to the ASTM standard, the acquired aluminium flat bars plates were cut by the Electrical Discharge Machining (EDM) machine according to the shape and measures shown in Figure 1. Three specimens were cut in directions $0^{\circ}, 45^{\circ}$ and $90^{\circ}$; the degrees are in relative to rolling direction.

The specimen surface to be studied must have a speckle pattern. First painting the surface with a narrow layer of white color and then applying a black mist of paint (spray paint) to create the black speckles. To apply the black mist of paint it is essential to keep approximate two feet of distance between the specimen and spray. Figure 2 illustrates a speckle pattern. 
Table 1. Error sources of 2D DIC [12].

\begin{tabular}{ll}
\hline Errors related to specimen, loading and imaging & Speckle pattern \\
& Nonparallel between the \\
& CCD target and the object \\
& Surface and out-of-plane dis- \\
& placement \\
& Imaging distortion \\
& Noises during image acquisi- \\
& tion and digitization \\
\hline Errors related to the correlation algorithm & Subset size \\
& Correlation function \\
& Sub-pixel algorithm \\
& Shape function \\
& Interpolation scheme \\
\hline
\end{tabular}

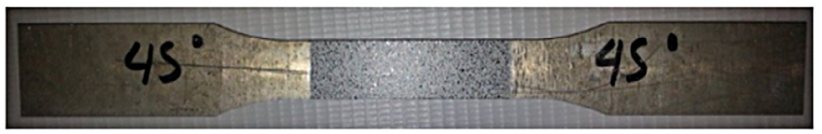

Fig. 2. Specimen with speckle pattern.

Table 2. Settings for the video camera.

\begin{tabular}{cc}
\hline Settings & Aluminium \\
\hline Aperture & F2.0 \\
Frame Rate & $25 \mathrm{fps}$ \\
Image resolution & $1920 \times 1080$ \\
\hline
\end{tabular}

\subsection{Sample loading and imaging}

Before the tensile test was started, it was necessary to make sure that the video camera was properly installed on top of the tripod. Since the tripod can be rotated in yaw, pitch and roll axis, a series of alignment checking were carried out to ensure that the video camera was installed correctly. A spirit level was placed on the body of the video camera and the alignments were adjusted to be flat in the axis of pitch and roll. Besides this, the specimen was vertically aligned too during the installation of the specimen into the middle of the UTM grippers. The spirit level was placed alongside the dog-bone shape specimen and the alignment of the specimen in the vertical axis was verified. Next, the extensometer was gently mounted on the specimen and the results obtained served as a benchmark for values determined from the optical strain measurement method.

After the alignment checking was done in the experimental setup, all the equipment such as the UTM grippers, the tripod, the video camera was kept untouched for the entire tensile test. Furthermore, the experimental setup discussed above is very suitable for aluminium specimen. Figure 3 shows the experimental setup.

After this, the UTM switched on and the cross head speed was set at the rate of $0.01 \mathrm{~mm} . \mathrm{s}^{-1}$. Meanwhile, the video camera was adjusted according to the setting for aluminium as in Table 2 .

The tensile test was conducted once the specimen and the extensometer were properly installed. Simultaneously,

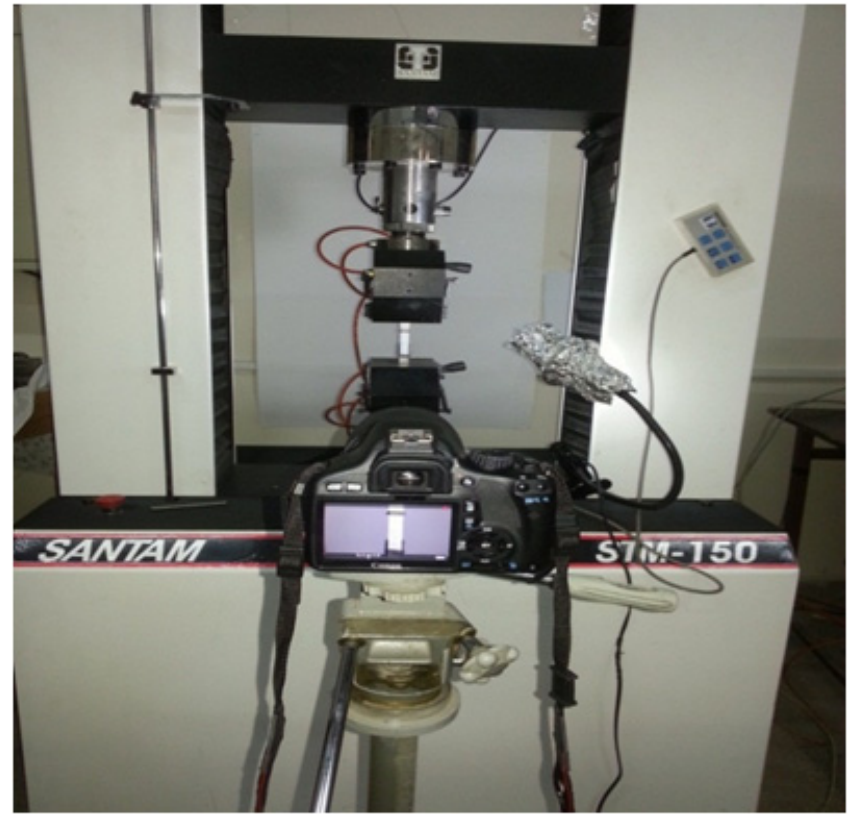

Fig. 3. Experimental setup.

the videos were recorded using a consumer version of high definition video camera at the rate of $25 \mathrm{fps}$. One experiment took an average of 6 to 8 min.

\subsection{Digital image correlation}

Here, to obtain displacements and strains, GUIs in MATLAB commercial program was used. The foundation of the correlation procedure is in a function named cpcorr_mod.m; This function is a slight modification of the MATLAB's standard function cpcorr.m, which can be found in toolbox of Image Processing. This algorithm assumes some pixels nearby the control point as a subset, in deformed image, and a larger subset (almost twice as large) around the exact control point in the reference image as illustrated in Figure 4; this process is done for every control point. The search zone adjusts the relationship of the subset size between reference and deformed image. 


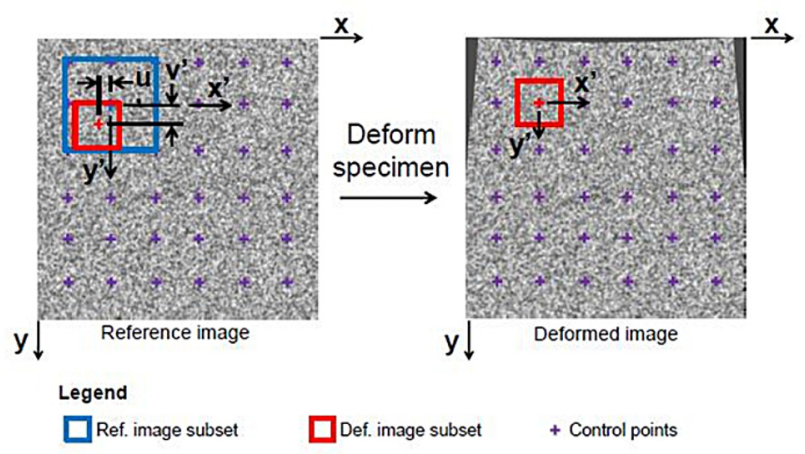

Fig. 4. Sketch of DIC model. A rectangular grid of pixels is applied to the region of concern as control points (purple crosses). The blue box shows the subset before deformation in the reference image and the red box shows the corresponding subset in the deformed state. The normalized cross correlation coefficient is evaluated from Equation (1). The maximum correlation coefficient will result in obtaining the real displacement $(u, v)$ [14].

Bigger search zone will result in bigger maximal acceptable displacement, yet at the expense of a wider boundary around the intended region in which correlation can't be applied to the control points.

The algorithm calculates the cross-correlation factor, $C$, in normalized form (Eq. (1)) for a domain of theoretic displacements, $\left(u_{0}, v_{0}\right)$, in 1 pixel increases by entwisting the subset from the deformed image with the wider subset from the reference image as stated in:

$$
\begin{aligned}
& C\left(u^{\prime}, v^{\prime}\right)= \\
& \frac{\sum_{x^{\prime}, y^{\prime}}\left[\left(r\left(x^{\prime}, y^{\prime}\right)-\bar{r}_{u^{\prime}, v^{\prime}}\right)\left(d\left(x^{\prime}-u^{\prime}, y^{\prime}-v^{\prime}\right)-\bar{d}\right)\right]}{\left\{\sum_{x^{\prime}, y^{\prime}}\left[\left(r\left(x^{\prime}, y^{\prime}\right)-\bar{r}_{u^{\prime}, v^{\prime}}\right)^{2}\right]_{x^{\prime}, y^{\prime}}\left[\left(d\left(x^{\prime}-u^{\prime}, y^{\prime}-v^{\prime}\right)-\bar{d}\right)^{2}\right]\right\}^{1 / 2}}
\end{aligned}
$$

wherein $r$ is the severity of the pixels in the reference subset, $d$ is the severity of the pixels in the deformed subset, and $\left(x_{0}, y_{0}\right)$ are positional subset coordinate axes; It's origin is at center of subset at the control point. To compute displacements in 1/100 of a pixel, 9 separate correlation factors surrounding the definite maximal factor are interpolated with the use of a polynomial in second order at $u_{0}$ and $v_{0}$. Accurate displacement of a control point is the theoric displacement $\left(u_{0}, v_{0}\right)$ relating to the interpolated correlation factor which is maximal. Every control point will have some discrete displacement values which come from the algorithm as output [14-16].

\subsection{Strain calculation}

In order to calculate strains, displacements should be interpolated with the help of shape functions in finite element form.

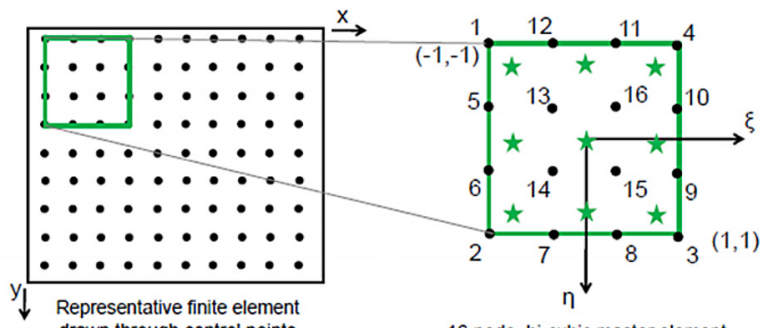

drawn through control points from image correlation

16-node, bi-cubic master element

Legend

$\square$ Finite element $\quad$ Displacement node $\star$ Quadrature point

Fig. 5. Sketch of FE model used to calculate strain. With knowledge of displacements at control points (black circles), a finite element with 16 nodes (green square) is assumed as illustrated. The element is mapped to another element called master, with local coordinates 5 and 5 ; The displacements are obtained by interpolating over the master element using bicubic shape functions. After interpolating displacements, the derivatives are evaluated at the nine Legendre-Gauss points (green stars), and finally mapped back to the original element [14].

Before extracting strains, we have to smooth displacements to supply resistance against the nature of noise in DIC. The boundaries of the region of concern must be padded to decrease side effects; To do this, displacement values at the border of the region of concern are reflected among the boundary so that continuity of the first derivative is satisfied.

An element with 16 nodes is assumed so that the control points in image correlation correspond to these nodes in finite element. Then the mapping function relates this element to the master element (Fig. 5) using:

$$
(x, y)=f^{i}(\xi, \eta)=\underline{\hat{N}}(\xi \eta) \underline{X}^{i}
$$

Wherein $(x, y)$ are the image coordinates, $(\xi, \eta)$ are the local coordinates of the master element, and $f^{i}$ is the function of mapping; mapping function is defined as multiplication (in matrix form) of the shape functions on the master element, $\underline{\hat{N}}$, and the nodes coordinate for element $i$ in image, $\underline{X}^{i}$. he shape functions for the above mentioned finite element, $\hat{N}_{j}$, would be a bicubic of sixteenth degree which is as follows:

$$
\begin{array}{r}
\hat{N}_{j}(\xi \eta)=\left(a \xi^{3}+b \xi^{2}+c \xi+d\right)\left(e \eta^{3}+f \eta^{2}+g \eta+h\right) \hat{N}_{j}(\xi, \eta) \\
=\left\{\begin{array}{l}
1 \quad \text { at node } \mathrm{j} \\
0 \text { at all other nodes }
\end{array}\right.
\end{array}
$$

Here $a, b, \ldots, h$ are the constants. Interpolation over the master element will result in displacements, then with the use of the relationship below they are translated back to the original element:

$$
\underline{U}^{I}(x, y) \|_{(x, y)=f^{i}(\xi, \eta)}=\underline{\hat{N}}(\xi \eta) \underline{U}_{E L}^{i}
$$

Here $\underline{U}^{I}(x, y)$ is a function of image coordinates $(x, y)$ which is interpreted as interpolated displacements and 
$\underline{U}_{E L}^{i}$ contains node's displacements for the " $i$ " th element in the directions of $x$ and $y \cdot \nabla U(x, y)$, the first derivatives (partial), of the interpolated displacements are evaluated using:

$$
\underline{\nabla U}(x, y) \|_{(x, y)=f^{i}(\xi, \eta)}=\underline{\nabla \hat{N}}(\xi \eta)\left[\underline{J}^{i}(\xi, \eta)\right]^{-1} \underline{U}_{E L}^{i}
$$

where in $\underline{J}^{i}$ is the Jacobian of the mapping function for element $i$, calculated through:

$$
\underline{J}^{i}(\xi, \eta)=\left[f^{i}(\xi, \eta)\right]^{\prime}=\underline{\nabla \hat{N}}(\xi \eta) \underline{X}^{i}
$$

Nine Legendre-Gauss points are found to be the most accurate points for interpolation algorithm on the master element, which are a mixture of $(\xi, \eta)=-\sqrt{\frac{3}{5}}, 0, \sqrt{\frac{3}{5}}$. Hence, the displacement derivatives are evaluated at these discontinuous points for all elements that can be possibly drawn between the control points. Gauss points which are overlapped from another neighbor elements, are averaged; presenting discontinuous values on the displacement gradient in a grid that is a little bit smaller than the grid of control points. At last, each component of the small strain tensor, $\epsilon_{i j}$ is computed from [14]:

$$
\epsilon_{i j}=\frac{1}{2}\left(\frac{\partial U_{i}}{\partial X_{j}}+\frac{\partial U_{j}}{\partial X_{i}}\right)
$$

\subsection{Measurement of $R$}

The value of $R$ is calculated as the ratio of width strain to thickness strain $[17,18]$; the thickness strain of a thin sheet cannot be measured accurately. Instead, the thickness strain is found from the width and length strains, $\varepsilon_{t}=-\left(\varepsilon_{w}+\varepsilon_{l}\right)$.

Usually each specimen cut from aluminium sheet in each individual direction $\left(0^{\circ}, 45^{\circ}\right.$ and $\left.90^{\circ}\right)$, before deformation, is meshed with circular grids using chemical methods, to obtain the coefficient of plastic anisotropy. Then all specimens are experimented under uniaxial tensile tests until the deformation enters in completely plastic area. After the tests are done, the circles turn into ellipses. Afterwards the two diameters of ellipses are measured with such tools as myler tape, optic microscope or etc.; So the axial and transverse strains $\left(\varepsilon_{l} \varepsilon_{w}\right)$ are calculated. With these data and the relation below, the coefficient of plastic anisotropy is calculated:

$$
r=\frac{\varepsilon_{w}}{\varepsilon_{t}}=\frac{\varepsilon_{w}}{-\left(\varepsilon_{w}+\varepsilon_{l}\right)}
$$

Notice that as the circular grids are created using chemical methods, some features of metal could change. Also, dimension measurements are done using Myler tape, optic microscope or etc. which are time consuming and with great errors. Regarding that in this research with DIC method, the largest axial and transverse displacements are obtained, the coefficient of plastic anisotropy could be calculated easily. One of the great advantages of this method is that even in a few seconds after tensile tests are begun, the failure of the specimen is predictable.

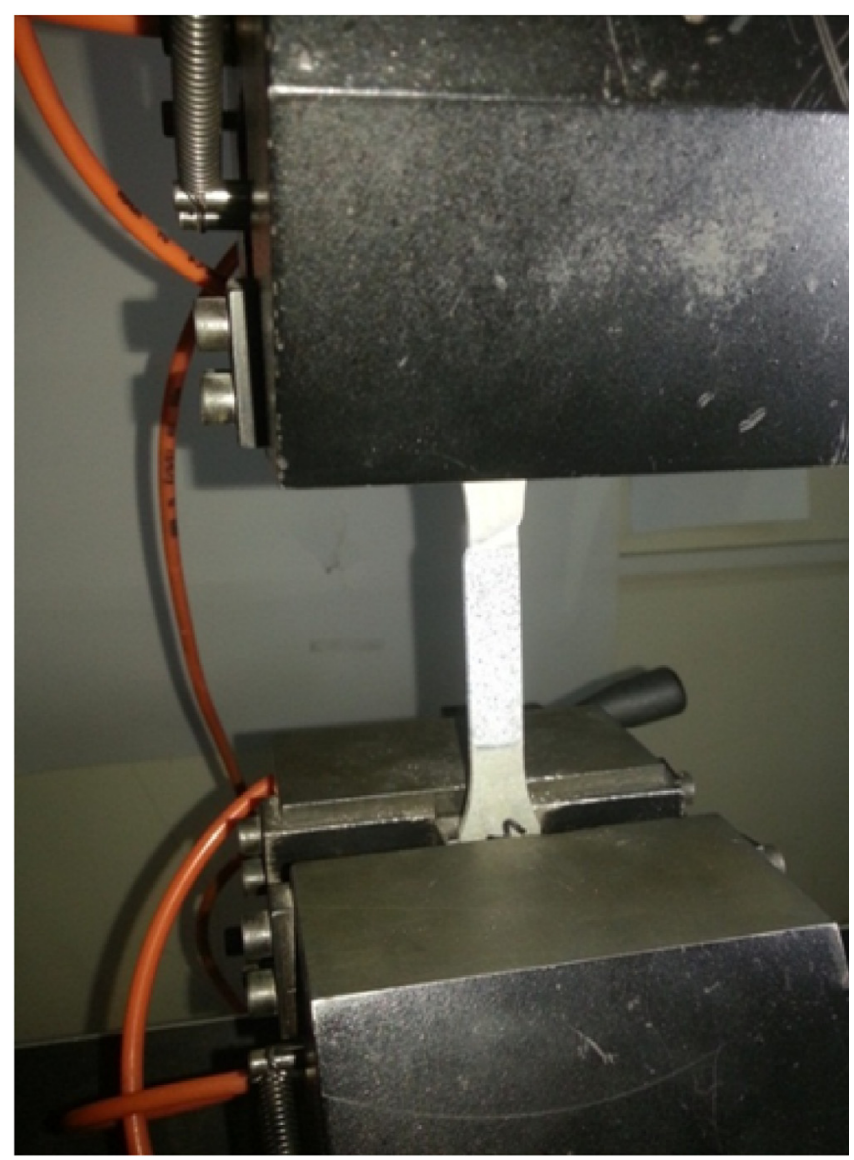

Fig. 6. Specimen failure after tensile test.

\section{Result and discussion}

As can be understood from the Figure 6, at the end of tensile test, specimen starts to fail from above. The displacement results obtained from DIC clearly shows that displacements in the upper area of specimen are larger than other areas.

\subsection{Displacement}

Figures 7-9 show the y displacements on surface of $0^{\circ}, 45^{\circ}, 90^{\circ}$ specimens at the time $120 \mathrm{~s}$, respectively. It is possible to observe the displacements at any other desired time of the test.

Figures 10-12 show the $x$ displacements on surface of $0^{\circ}, 45^{\circ}, 90^{\circ}$ specimens at the time $120 \mathrm{~s}$, respectively. It is possible to observe the displacements at any other desired time of the test.

Figures 13-15 show the average $y$ displacements over the entire image of $0^{\circ}, 45^{\circ}, 90^{\circ}$ specimens respectively.

Figures $16-18$ show the average $x$ displacements over the entire image of $0^{\circ}, 45^{\circ}, 90^{\circ}$ specimens respectively.

To verificate displacement results, these data were compared to extensometer data. Figures 19-21 show this comparison for $0^{\circ}, 45^{\circ}, 90^{\circ}$ specimens, respectively. 
N. Hedayati et al.: Mechanics \& Industry 18, 311 (2017)

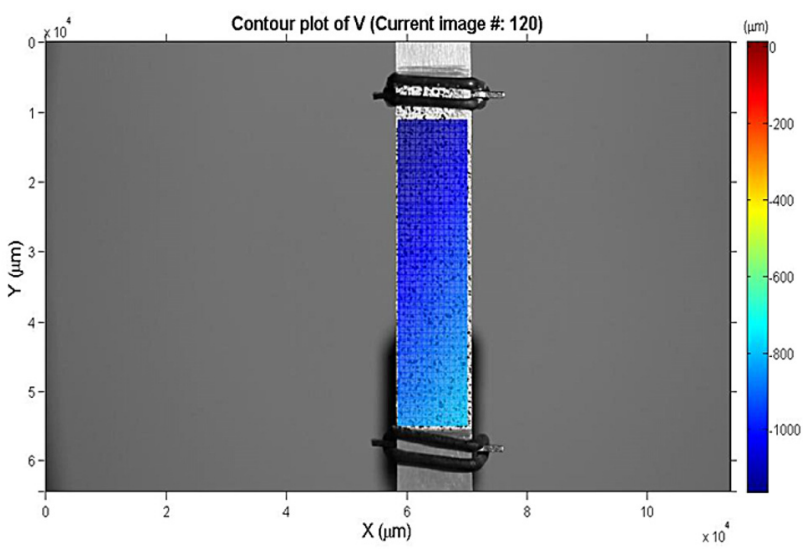

Fig. 7. $y$ displacements for $0^{\circ}$ specimen at the time 120 s.

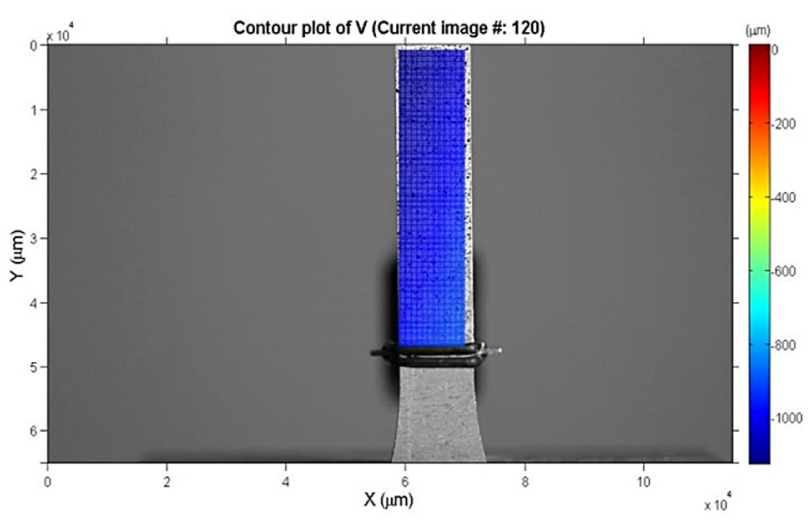

Fig. 8. $y$ displacements for $45^{\circ}$ specimen at the time 120 s.

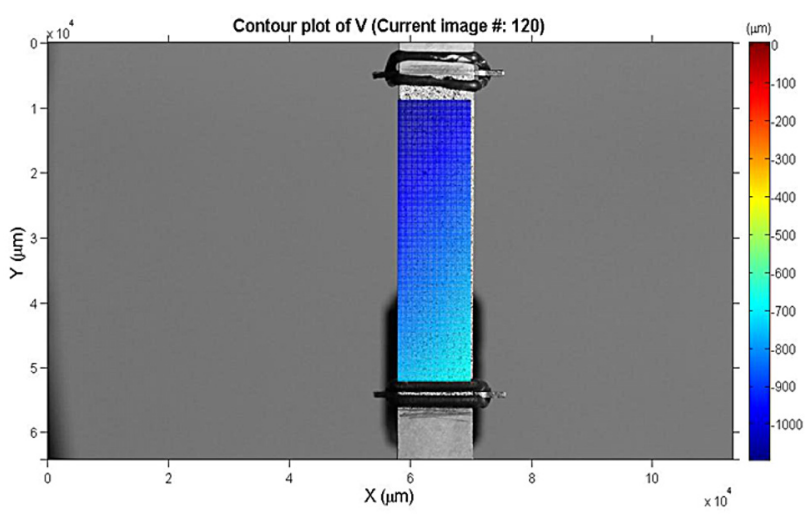

Fig. 9. $y$ displacements for $90^{\circ}$ specimen at the time 120 s.

Table 3 shows error between displacement obtained from DIC and extensometer data at the moment 120s for each 3 specimens.

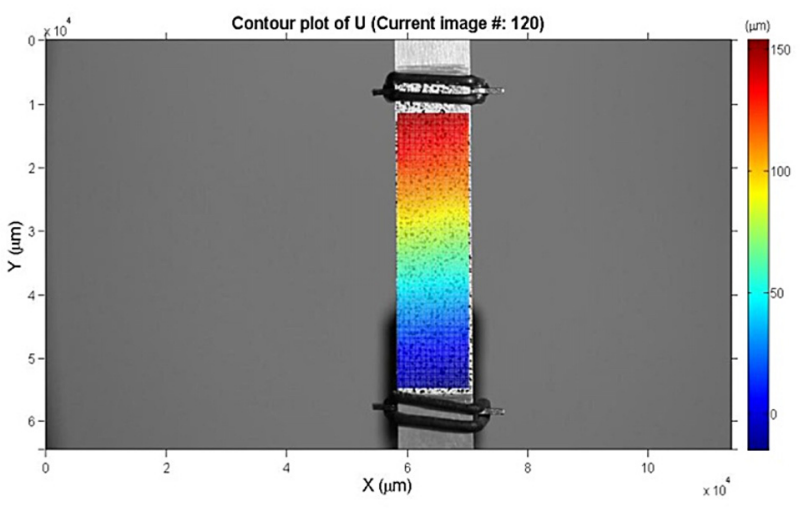

Fig. 10. $x$ displacements for $0^{\circ}$ specimen at the time 120 s.

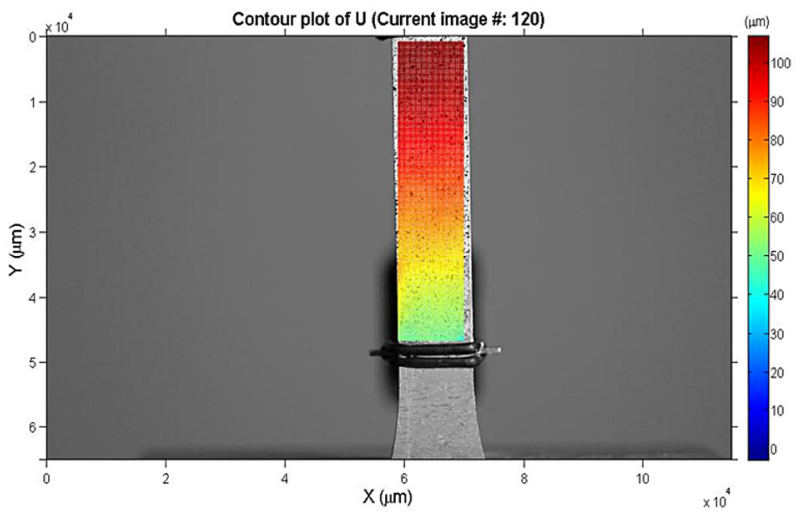

Fig. 11. $x$ displacements for $45^{\circ}$ specimen at the time 120 s.

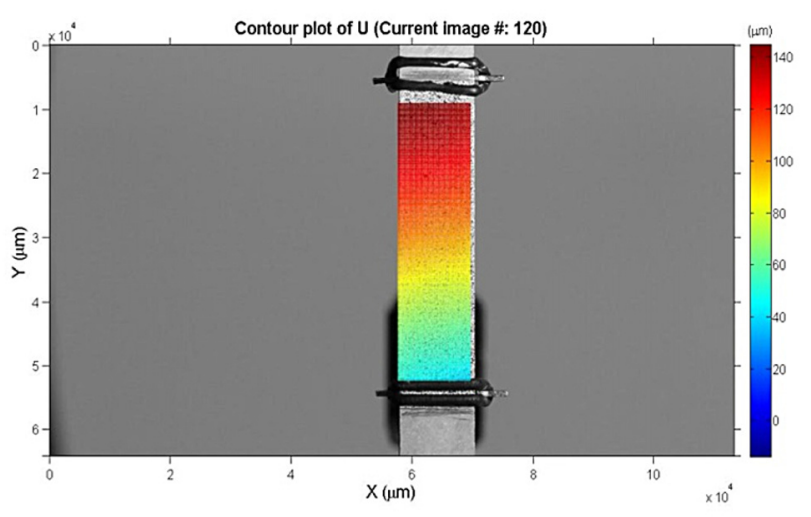

Fig. 12. $x$ displacements for $90^{\circ}$ specimen at the time 120 s.

Table 3. Error between DIC and extensometer data.

\begin{tabular}{ccc}
\hline DIC $(\mathrm{mm})$ & Extensometer $(\mathrm{mm})$ & Error $(\%)$ \\
\hline 1.165 & 1.1995 & 2 \\
1.126 & 1.1994 & 6 \\
1.095 & 1.1997 & 8 \\
\hline
\end{tabular}


N. Hedayati et al.: Mechanics \& Industry 18, 311 (2017)

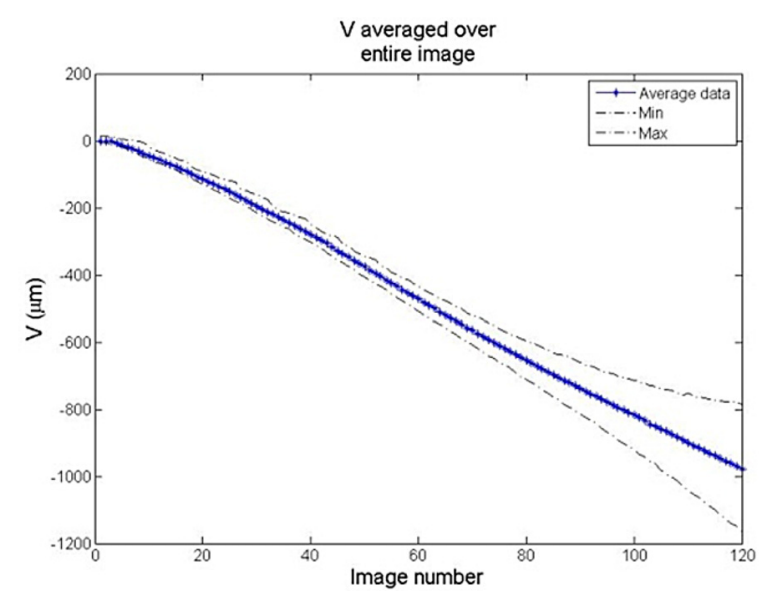

Fig. 13. Average $y$ displacements over entire image of $0^{\circ}$ specimen.

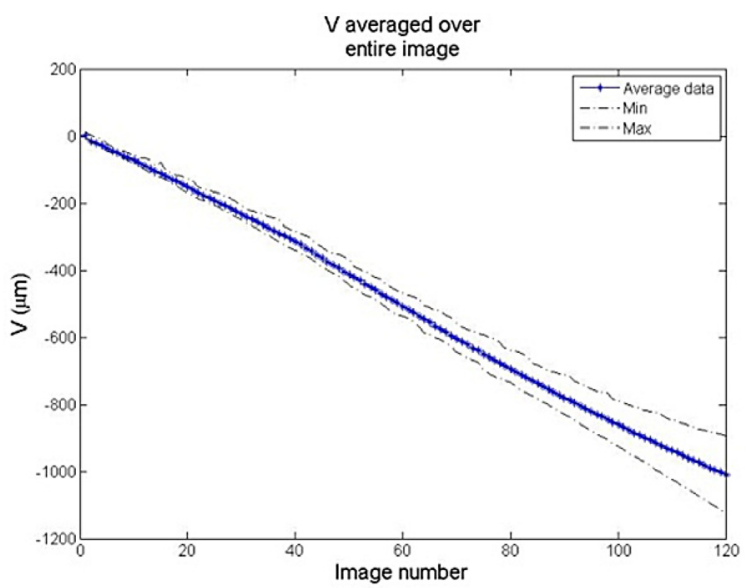

Fig. 14. Average $y$ displacements over entire image $45^{\circ}$ specimens.

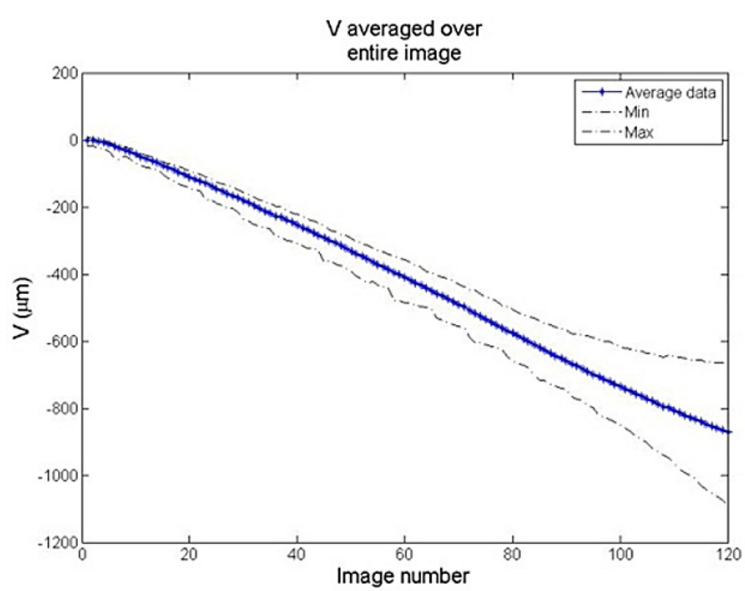

Fig. 15. Average $y$ displacements over entire image $90^{\circ}$ specimens.

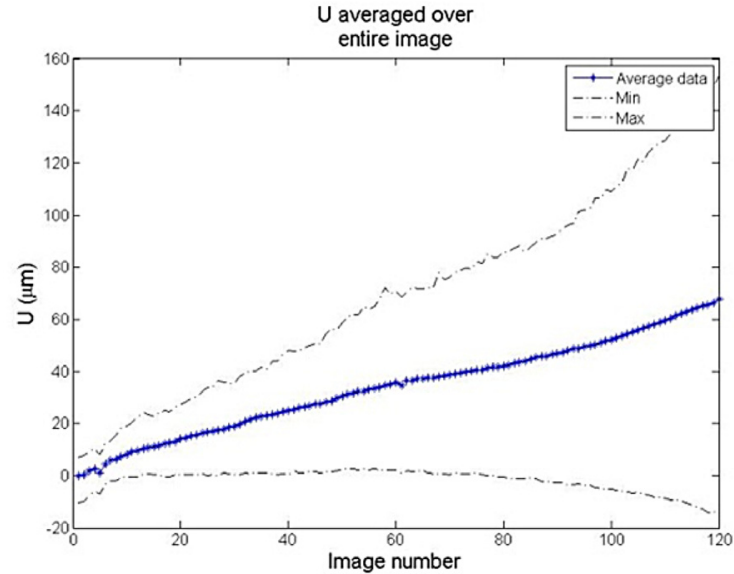

Fig. 16. Average $x$ displacements over entire image $0^{\circ}$ specimens.

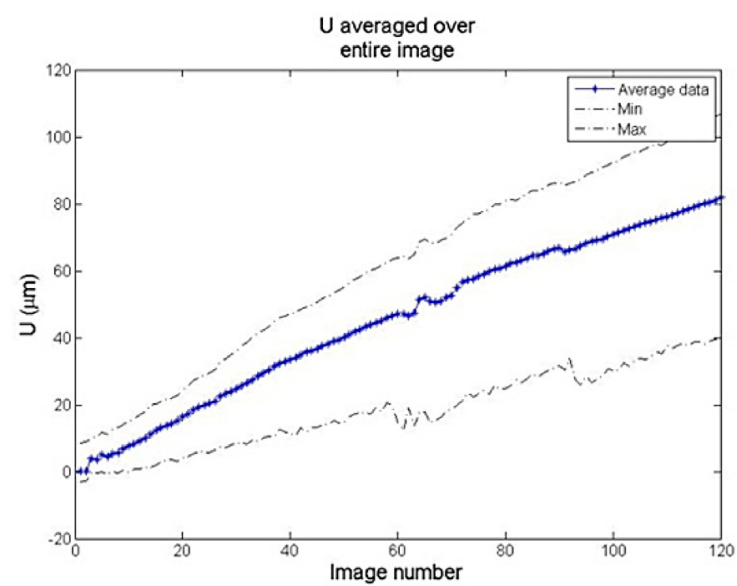

Fig. 17. Average $x$ displacements over entire image $45^{\circ}$ specimens.

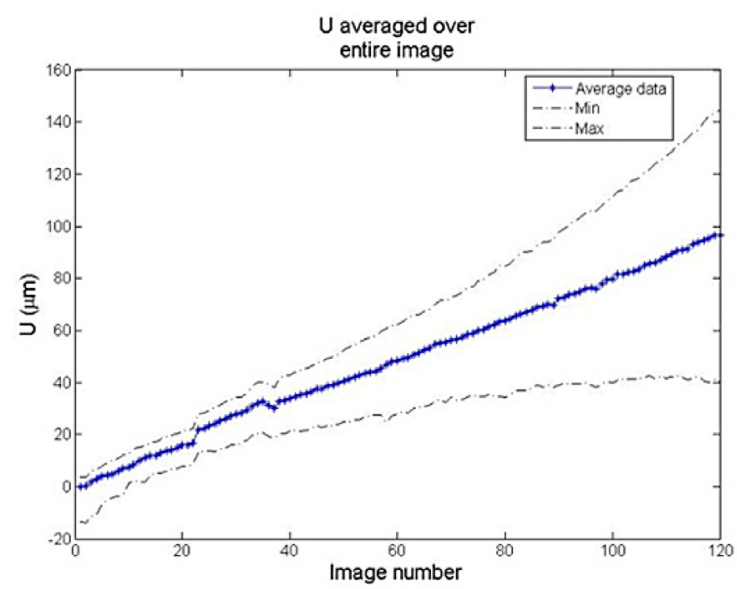

Fig. 18. Average $x$ displacements over entire image $90^{\circ}$ specimens. 


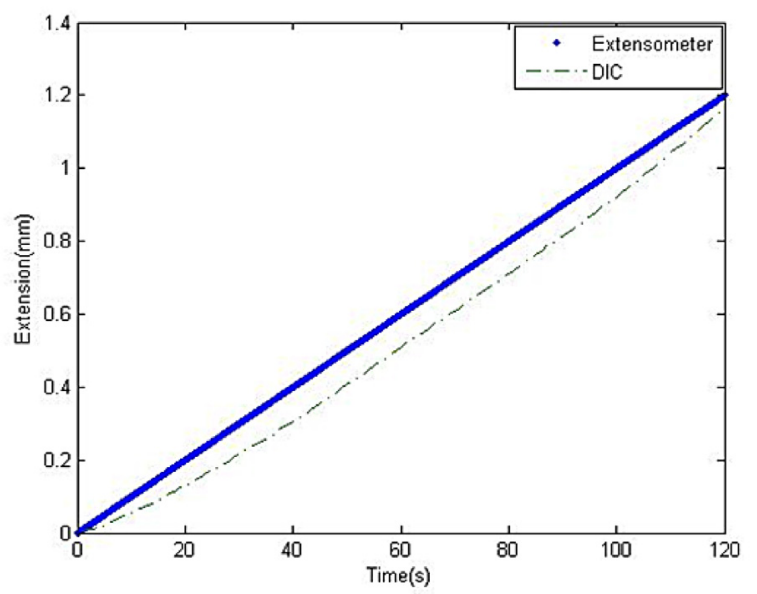

Fig. 19. Comparison of extensometer data with DIC for $0^{\circ}$ specimen.

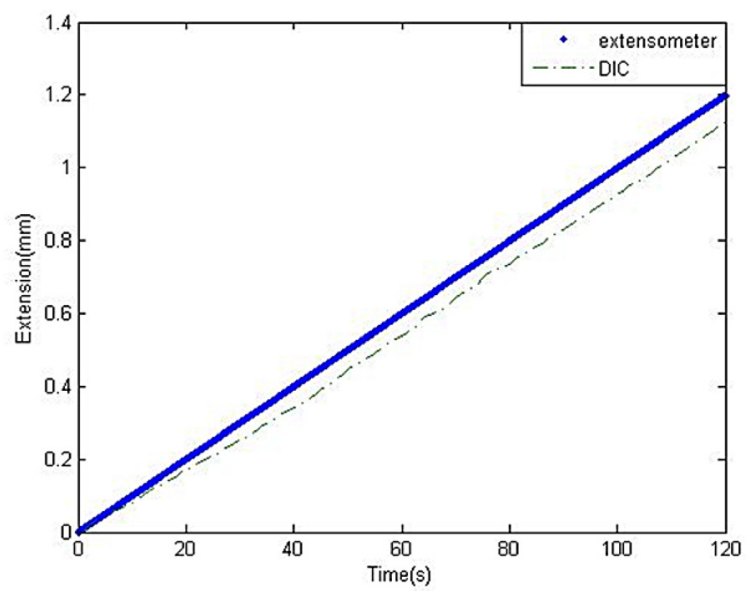

Fig. 20. Comparison of extensometer data with DIC for $45^{\circ}$ specimen.

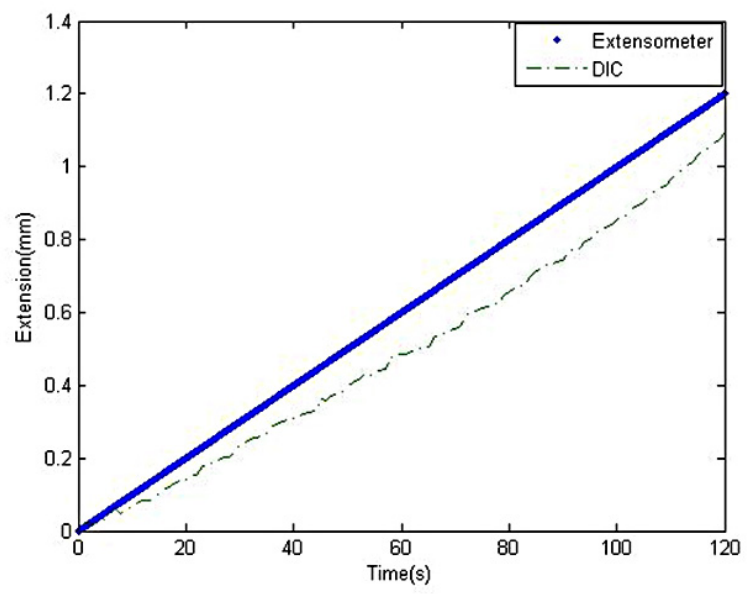

Fig. 21. Comparison of extensometer data with DIC for $90^{\circ}$ specimen.

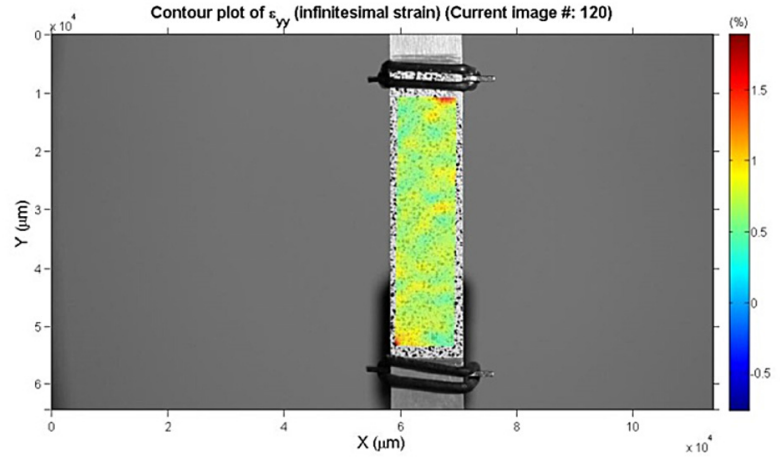

Fig. 22. $\epsilon_{y y}$ for $0^{\circ}$ specimen.

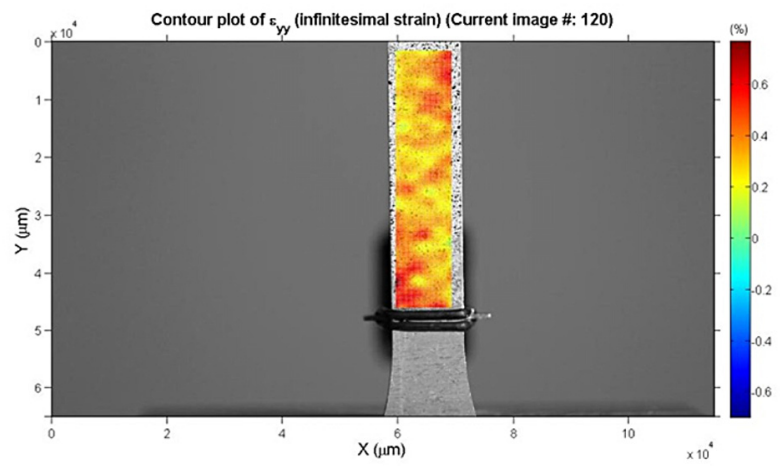

Fig. 23. $\epsilon_{y y}$ for $45^{\circ}$ specimen.

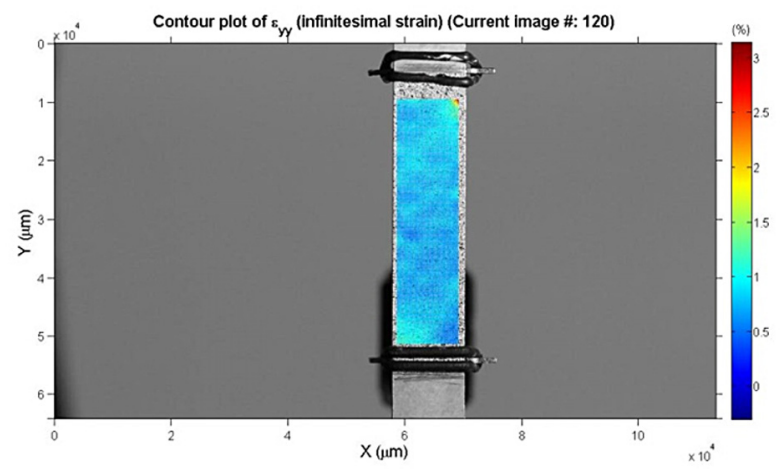

Fig. 24. $\epsilon_{y y}$ for $90^{\circ}$ specimen.

\subsection{Strain}

Figures 22-24 illustrates $\epsilon_{y y}$ for $0^{\circ}, 45^{\circ}, 90^{\circ}$ specimens, respectively.

Figures 25-27 illustrates $\epsilon_{x x}$ for $0^{\circ}, 45^{\circ}, 90^{\circ}$ specimens, respectively.

\subsection{R-value}

Since the displacement results from DIC are nearly in accordance with extensometer data, so the coefficient of plastic anisotropy can be calculated.

To obtain the coefficient of plastic anisotropy, the largest displacement in specimen should be determined. 
N. Hedayati et al.: Mechanics \& Industry 18, 311 (2017)

Table 4. Geometrical characteristics of the specimens.

\begin{tabular}{cccccc}
\hline Angle & $y$ displacement $(\mathrm{mm})$ & $x$ displacement $(\mathrm{mm})$ & Gauge length $(\mathrm{mm})$ & Width $(\mathrm{mm})$ & R-value \\
\hline $0^{\circ}$ & 1.164 & 0.1517 & 50 & 12.61 & 1.1093 \\
$45^{\circ}$ & 1.125 & 0.1063 & 50 & 12.61 & 0.6141 \\
$90^{\circ}$ & 1.091 & 0.144 & 50 & 12.58 & 1.143 \\
\hline
\end{tabular}

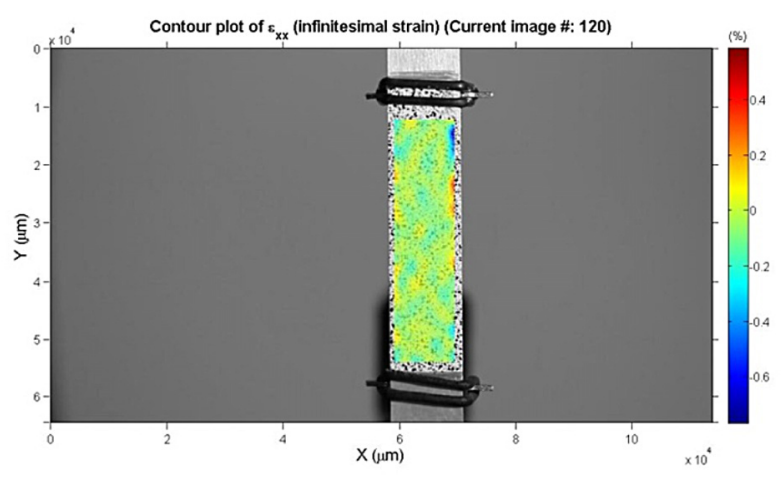

Fig. 25. $\epsilon_{x x}$ for $0^{\circ}$ specimen.

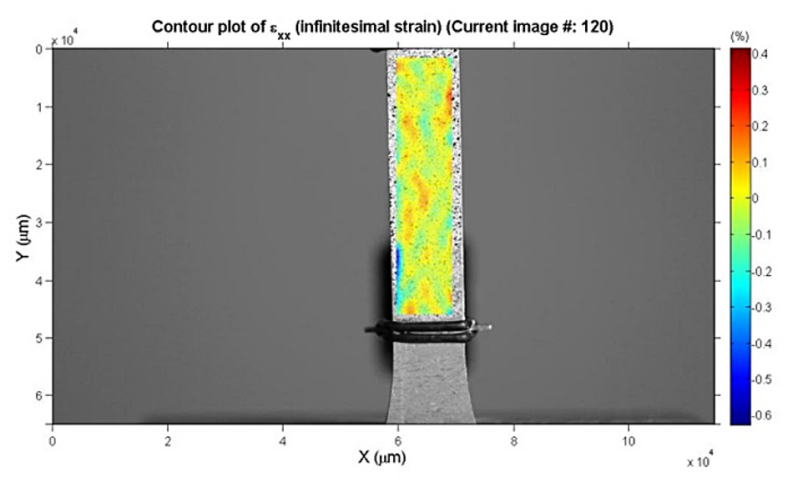

Fig. 26. $\epsilon_{x x}$ for $45^{\circ}$ specimen.

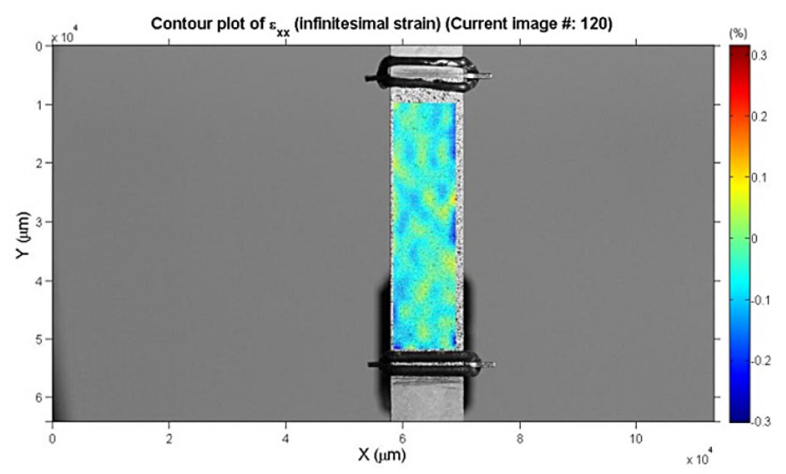

Fig. 27. $\epsilon_{x x}$ for $90^{\circ}$ specimen.

Since the largest displacement occurs in the upper side of the specimen, so the largest displacement associated with that area must be determined and then axial and transverse strains will be calculated according to this data; At the end, coefficient of plastic anisotropy will be calculated.

Table 4 shows the coefficients of plastic anisotropy determined from the DIC method for $0^{\circ}, 45^{\circ}, 90^{\circ}$ specimens.

\section{Conclusion}

Research over the last two decades made DIC an effective and flexible method for measuring surface displacements and strains in macro, micro and even nano scales. This technique is an optical method which makes it classified as an undestructive one. This method has been used in several wide fields successfully and proved that it is a powerful and useful tool for determining deformations; yet it is going to gain more attention in scientific communities.

By comparing the results determined by the extensometer and the optical strain measurement method, the deviations in terms of percentage had been found to be relatively small. In conclusion, the two-dimensional measurement algorithm that calculates the strain in a loaded structural had been developed successfully and tested in the study. In addition, the consumer version of highdefinition video camera has been proven to be capable to capture the deformed images impeccably. Since special softwares for DIC method such as Vic are expensive, so using DIC method with MATLAB commercial program would be an economical choice.

\section{References}

[1] A. Giachetti, Matching techniques to compute image motion, Image Vis. Comput 18 (2000) 247-260

[2] W. Tong, An evaluation of digital image correlation criteria for strain mapping applications, Strain 41 (2005) $167-175$

[3] K. Triconnet, K. Derrien, F. Hild, D. Baptiste, Parameter choice for optimized digital image correlation, Opt. Lasers Eng. 47 (2009) 728-237

[4] W.H. Peters, W.F. Ranson, Digital imaging techniques in experimental stress analysis, Opt. Eng. 21 (1982) 427-431

[5] M.A. Sutton, W.J. Wolters, W.H. Peters, W.F. Ranson, S.F. McNeill, Determination of displacements using an improved digital correlation method, Image Vis. Comput. 1 (1983) 133-139

[6] D.J. White, W.A. Take, M.D. Bolton, Soil deformation measurement using particle image velocimetry (PIV) and photogrammetry, Géotechnique 50 (2003) 619-631

[7] R.W. BrachmanI, H.A. McLeod, I.D. Moore, W.A. Take, Three-dimensional ground displacements from static pipe bursting in stiff clay, Can. Geotech. J. 47 (2010) 439-450

[8] M.A. Sutton, J.-J. Orteu, H.W. Schreier, Image correlation for shape, motion and deformation measurements, Springer Science + Business Media, New York, 2009 
[9] B.W. Smith, X. Li, W. Tong, Error assessment for strain mapping by digital image correlation, Exp. Tech. 22 (1998) 19-21

[10] B. Wattrisse, A. Chrysochoos, J.-M. Muracciole, NémozGaillard M. Analysis of strain localization during tensile tests by digital image correlation, Exp. Mech. 41 (2001) 29-39

[11] P.C. Hung, A.S. Voloshin, In-plane strain measurement by digital image correlation, J. Braz. Soc. Mech. Sci. Eng. 25 (2003) 215-221

[12] P. Bing, Q. kemoa, A. Anand, X. Humin, Twodimensional digital image correlation for in-plane displacement and strain measurement: a review, Meas. Sci. Technol. 20 (2009) 062001

[13] H. Haddadi, S. Belhabib, Use of rigid-body mOtion for the investigation and estimation of the measurement errors related to digital image correlation technique, Opt. Lasers Eng. 46 (2008) 185-196
[14] http://www.mathworks.com/ matlabcentral/fileexchange/ 43073-improved-digital-image-correlation--dic- , (2013)

[15] N.A. Hoult, W.A. Take, Lee C, Dutton M. Experimental accuracy of two dimensional strain measurements using digital image correlation, Eng. Struct. 46 (2013) 718-726

[16] G. Crammond, S.W. Boyd, J.M. Dulieu-Barton, Speckle pattern quality assessment for digital image correlation, Opt. Lasers Eng. 51 (2013) 1368-1378

[17] W.F. Hosford, R.M. Caddell, Metal Forming: Mechanics and Metallurgy 4th Edition, Cambridge University Press, 2014

[18] H. Mamusi, A. Masoumi, R. Hashemi, R. Mahdavinejad, A novel approach to the determination of forming limit diagrams for tailor-welded blanks, J. Mater. Eng. Performance 22 (2013) 3210-3221 